\title{
Mondes du Tourisme
}

Jérémy Lemarié, Genèse d'un système global surf. Regards comparés des Hawai'i à la Californie : traditions, villes, tourismes, et subcultures (1778-2016)

Thèse de doctorat en sociologie de l'université Paris-Ouest Nanterre La Défense, dirigée par Jonathan Friedman et Anne Raulin (soutenue le 20 mai 2016).

Jérémy Lemarié

\section{OpenEdition}

Édition électronique

URL : http://journals.openedition.org/tourisme/1331

DOI : 10.4000/tourisme. 1331

ISSN : 2492-7503

Éditeur

Éditions touristiques européennes

Référence électronique

Jérémy Lemarié, « Jérémy Lemarié, Genèse d'un système global surf. Regards comparés des Hawai'i à la Californie : traditions, villes, tourismes, et subcultures (1778-2016)», Mondes du Tourisme [En ligne], 12 |

2016, mis en ligne le 01 décembre 2016, consulté le 23 septembre 2020. URL : http://

journals.openedition.org/tourisme/1331 ; DOI : https://doi.org/10.4000/tourisme.1331

Ce document a été généré automatiquement le 23 septembre 2020.

Mondes du tourisme est mis à disposition selon les termes de la licence Creative Commons Attribution - Pas d'Utilisation Commerciale - Pas de Modification 4.0 International. 


\section{Jérémy Lemarié, Genèse d'un système global surf. Regards comparés des Hawai'i à la Californie : traditions, villes, tourismes, et subcultures (1778- 2016)}

Thèse de doctorat en sociologie de l'université Paris-Ouest Nanterre La Défense, dirigée par Jonathan Friedman et Anne Raulin (soutenue le 20 mai 2016).

Jérémy Lemarié

\section{RÉFÉRENCE}

Jérémy Lemarié, Genèse d'un système global surf. Regards comparés des Hawai'i à la Californie : traditions, villes, tourismes, et subcultures (1778-2016), thèse de doctorat en sociologie, université Paris Ouest Nanterre La Défense, 2016.

1 À partir de l'histoire coloniale des Hawai'i et des États-Unis en 1778, cette recherche traite de la transformation de la coutume hawaïenne he'e nalu en un "système surf mondial ». Le travail analyse les modalités de diffusion et de réappropriation de la culture hawaïenne vers l'Occident et s'interroge sur les structures politiques, économiques et idéologiques qui ont permis la métamorphose progressive d'une pratique autochtone en un sport actuellement pratiqué par trente millions de personnes dans plus d'une centaine de pays. La plupart des études sur le sujet ont souvent déconnecté le surf «moderne » de ses racines indigènes. Le hé $e$ nalu aurait quasiment disparu et aurait ressurgi au début du $\mathrm{xx}^{\mathrm{e}}$ siècle avec la création de l'Outrigger Canoe Club en 1908 et celle du Hui Nalu en 1911 à Waikîkī (Finney et Houston, 1996). Or, un récent courant de pensée défend l'existence 
d'une continuité culturelle (Clark, 2011; Moser, 2016; Walker, 2011). Notre problématique tente de dépasser ces deux théories contradictoires. En quoi y a-t-il rupture et continuité du surf hawaïen au $\mathrm{XIX}^{\mathrm{e}}$ siècle, et quelles sont les modalités de sa transformation en un système global surf au $\mathrm{XxI}^{\mathrm{e}}$ siècle?

2 Pour répondre à la problématique, un travail d'archives a été effectué, reposant sur l'analyse des journaux de bord des voyageurs au XIX ${ }^{\mathrm{e}}$ siècle vers l'archipel des Hawai'i. Ces documents comprennent, entre autres, les carnets de James Cook, James King, David Samwell et George Vancouver. L'auteur a également consulté les chroniques de missionnaires comme celles d'Hiram Bingham et de William Ellis, ainsi que les textes d'écrivains, tels que les récits de Mark Twain ou de Charles Warren Stoddard. Ces témoignages ont été comparés avec une quinzaine d'articles de presse, et des recueils rédigés en hawaïen et en anglais, au sein desquels on identifie les écrits des premiers historiens de l'archipel comme ceux de Samuel Kamakau, Zephrin Kepelinio, David Malo et John Papa'I'î. Pour obtenir une vision d'ensemble, l'auteur a appris l'hawaïen durant deux ans au sein de l'association France-Hawai'i à Paris, ainsi qu'à l'université d'Hawai'i à Mānoa à l'automne 2015. Ensuite, cinquante entretiens semi-directifs ont été conduits aux Hawai'i et en Californie auprès d'acteurs politiques et économiques, pour saisir les enjeux de la réappropriation états-unienne du surf après 1945. L'échantillon se compose de femmes et d'hommes âgés de dix-huit à soixante-dix-huit ans, qui ont été interrogé selon la méthode du "sondage boule de neige». Afin de dégager les modalités contemporaines de l'historicisation du surf hawaïen, une observation participante multisite de trente mois à Honolulu, Huntington Beach et San Diego a également été réalisée entre 2009 et 2016. L'auteur a observé, et participé au quotidien de clubs de surf, d'associations bénévoles, et a visité les ateliers d'artisans-shapers. Une démarche itérative, consistant à réaliser des allers-retours entre le terrain et le lieu de résidence, a permis d'approfondir le degré d'immersion dans la culture étudiée, et de passer d'une enquête par immersion (etic) à une enquête par distanciation (emic).

3 Le premier chapitre de la thèse commence par une présentation du surf au sein des structures sociales hawaïennes avant l'arrivée des Occidentaux, et le deuxième chapitre montre pourquoi les missionnaires calvinistes et leur doctrine n'ont pas été les seuls responsables de la chute de la pratique du surf au XIX siècle (Lemarié, 2015, 2016). Naturellement, l'importation de maladies étrangères et la diminution dramatique de la population sont des facteurs déterminants, mais il faut également prendre en compte l'introduction des Hawai'i dans le commerce transpacifique du bois de santal, des baleiniers et de la fourrure qui ont transformé l'économie de subsistance de la société hawaïenne en une économie marchande. À cela s'ajoute le succès de nombreuses pratiques étrangères à Hawaï comme l'apprentissage de la lecture et de l'écriture, et l'utilisation généralisée des chevaux comme nouveau moyen de transport, qui a remplacé l'usage de la marche à pied et du déplacement en pirogue le long des côtes.

4 Après l'étude des principales causes du déclin du he`e nalu, cette recherche a montré au troisième chapitre que de nombreuses chroniques ont contredit l'idée que le surf hawaïen était en grande perdition. D'abord, les missionnaires constataient que cette coutume était toujours pratiquée plusieurs décennies après leur arrivée. Puis, les chirurgiens et physiciens à bord des navires ont défini le he'e nalu et le bain marin comme des pratiques revigorantes et nécessaires au maintien de l'hygiène. Aux côtés de cette vision hygiéniste importée depuis l'Europe, le regard romantique a également été déterminant pour 
attester de la vivacité du surf hawaïen, notamment grâce aux récits de voyage des premiers touristes comme Mark Twain, ou Charles Warren Stoddard.

5 Au quatrième chapitre, la recherche a étudié le point de vue indigène et s'est penchée sur la presse hawaïenne, très féconde entre les années 1860 et 1920. Contrairement à certains récits occidentaux, les textes hawaïens narrant le déclin du he'e nalu ont été rares durant cette période. Ce déclin est d'abord provenu d'un recul volontaire de la pratique, qui était de moins en moins populaire au regard des nouveaux sports importés par les Occidentaux, comme l'équitation qui a connu un succès fulgurant. Puis, la lecture et l'écriture ont été très prisées par les Hawaïens, or l'apprentissage dans les écoles se faisait surtout le matin, au moment où la pratique du surf était particulièrement répandue. L'alphabétisation a notamment permis d'établir un système administratif et juridique, ainsi que d'enregistrer par écrit l'histoire des indigènes. Puis, il faut aussi prendre en compte le contexte géopolitique du XIx ${ }^{e}$ siècle, c'est-à-dire l'émergence des États-nations. Avec la montée des nationalismes en Europe, la royauté hawaïenne avait compris l'importance d'affirmer ses traditions afin de forger une identité nationale indigène et de renforcer son poids diplomatique sur l'échiquier international. Le he'e nalu, tout comme le hula, est rapidement devenu une des spécificités culturelles de la société hawaïenne.

Avec l'annexion de l'archipel par les États-Unis en 1898 et l'avènement du tourisme balnéaire au $\mathrm{xx}^{\mathrm{e}}$ siècle, les Hawai'i se sont imposées comme un modèle touristique, reposant sur la mise en scène et la réappropriation occidentale de la culture polynésienne. Alors que la majorité des précédentes études s'accorde pour identifier Waikīīi comme le point de départ touristique du surf dans les années 1900, ce travail a démontré que c'est avant tout Hilo qui était le centre touristique du he'e nalu dans les années 1870. Cela s'explique principalement par les attractions que l'on visitait à partir d'Hilo, comme le cratère du volcan Kîlauea, et la vallée de Waipio.

7 Dans le cinquième chapitre, la recherche montre que la transformation du hée nalu en surf s'est également réalisée avec l'avènement des sports modernes en Angleterre au XIX ${ }^{\mathrm{e}}$ siècle. En effet, le modèle anglo-saxon qui a normé de nombreux jeux européens par l'instauration de codes, de règles et de clubs a été exporté jusqu'aux Hawai' $i$, puisque la transformation du hée nalu en surf s'est réalisée par l'intermédiaire de trois clubs : le Hui Pākākā Nalu, l'Outrigger Canoe Club, et le Hui Nalu. Bien que ces clubs aient plus été des concessions touristiques que des organisations sportives au sens strict, le Hui Nalu a popularisé la branche sportive du surf par l'intermédiaire de ses autochtones renommés, comme Duke Kahanamoku. Le surf s'est exporté en Australie et en Californie d'une part parce que les surfeurs hawaïens se conformaient à un idéal sportif masculin, et d'autre part parce qu'ils se conformaient à l'idéologie moderne grâce à l'application des techniques de navigation hawaïenne au sauvetage en mer au début du $\mathrm{xx}^{\mathrm{e}}$ siècle. L'utilisation de la planche de surf comme un outil essentiel du sauvetage en mer et la généralisation du métier de sauveteur ont grandement contribué à donner une image professionnelle du surf, qui a résonné avec les valeurs de la modernité, telles que la rigueur, le dévouement, l'entraînement sportif et l'hygiène physique.

8 Aux côtés de ce mouvement idéologique, les sixième et septième chapitres affirment qu'un mécanisme urbain a été à l'œuvre dans la métamorphose du he'e nalu en surf. En effet, des «scènes surf » ont émergé à partir des plages urbaines, et Waikikī constitue de ce point de vue un cas d'école. À l'instar de cette cité balnéaire hawaïenne, de nombreuses autres stations californiennes ont investi le surf comme un élément essentiel de leur identité territoriale, telles que Santa Cruz, Malibu, Santa Monica, Redondo Beach, 
Huntington Beach, San Clemente, San Onofre et San Diego. Avec une étude de cas comparés entre Waikīkī et Huntington Beach "Surf City USA ", la recherche a montré que ces deux stations partagent certaines similitudes structurelles et conjoncturelles dans leur développement balnéaire, comme l'adoption d'une économie touristique, l'établissement d'un service de plage et d'équipements de baignade, la présence de compétitions sportives et la mise en œuvre d'un plan de rénovation urbain suivant le thème du surf. Le septième chapitre démontre l'importance des scènes surf locales, et leur unification en une subculture surf transnationale par l'entretien de flux migratoires et touristiques entre stations et par la création de médias de niche comme le magazine Surfer et les films de surf tels que The Endless Summer.

Alors que la thèse reposait sur une dimension historique et chronologique, le huitième chapitre introduit les trente mois de terrain de recherche multi-site effectués par l'auteur, et les enjeux contemporains du « tourisme surf ». En lien avec son expérience en Californie et aux Hawai' $i$, l'auteur traite au neuvième chapitre de la professionnalisation du surf par le Windansea Surf Club de San Diego et par le circuit professionnel sur la côte nord de l'île d'O'ahu. L'analyse montre que le monde du surf a interagi avec des sponsors et des entreprises privés, et a spectacularisé la pratique par l'intermédiaire des médias en vue d'une reconnaissance sportive légitime par-delà son marché de niche. En réponse à l'intégration des logiques de marché dans le monde du surf, une branche dite "postmoderne " s'est construite avec la contre-culture des années 1970. Le surf post-moderne, encore appelé soul surfing, s'est opposé à la plupart des schémas traditionnels de la modernité, tels que la domestication des individus dans la sphère familiale, les systèmes spéculatifs et la société de consommation. Par la promotion du voyage de surf, que l'on appelle surf trip ou encore surfari, la diffusion du surf à l'international a reposé en partie sur le tourisme d'aventure.

Enfin, le dixième chapitre traite de l'utilisation de l'imagerie surf dans le secteur public et privé auprès des sites qui ont capitalisé sur cette ressource en tant qu'outil essentiel du développement touristique et du réaménagement urbain. Dans cette logique, Waikīkī et le centre-ville d'Huntington Beach sont les deux stations balnéaires à investir le plus l'image du surf et ses origines. Au regard des similarités entre ces deux stations, qui ont été comparées avec d'autres exemples comme Biarritz en France (Guibert, 2006) et Wanning en Chine (Guibert et Taunay, 2013), la recherche a dressé un modèle théorique qui détaille six stades de développement d'une station surf. Le caractère systématique d'un tel modèle révèlerait l'existence d'un système global, et la thèse a terminé son analyse par un appel à la recherche pour circonscrire plus finement l'histoire et les contours d'un tel système surf.

\section{BIBLIOGRAPHIE}

John R. K. Clark, Hawaiian Surfing: Traditions from the Past, University of Hawai'i Press, 2011.

Ben R. Finney et James D. Houston, Surfing: A History of the Ancient Hawaiian Sport, Pomegranate Artbooks, 1996. 
Christophe Guibert, L'Univers du surf et stratégies politiques en Aquitaine, L'Harmattan, 2006.

Christophe Guibert et Benjamin Taunay, « From political pressure to cultural constraints: The prime dissemination of surfing in Hainan », Journal of China Tourism Research, vol. 9, n 3, 2013, p. $365-380$

Jérémy Lemarié, « Debunking the myth that missionaries nearly killed surfing in the 19th century Hawai'i: Sandalwood, calvinists, germs and horses ", Trim, $\mathrm{n}^{\circ}$ 4, 2015, p. 44-68.

Jérémy Lemarié, « Débattre des performances culturelles hawaïennes au XIX siècle », Journal de la Société des océanistes, n 142-143, 2016, p. 159-174.

Patrick Moser, «The endurence of surfing in $19^{\text {th }}$ century Hawai' $i$ », Journal of the Polynesian Society, vol. 125, $\mathrm{n}^{\circ}$ 4, 2016, p. 411-432.

Isaiah H. Walker, Waves of Resistance: Surfing and History in Twentieth Century Hawai'i, University of Hawai'i Press, 2011. 